\title{
Predictive role of interleukin-6 and CAT score in mechanical ventilation in patients with chronic obstructive pulmonary disease at the acute exacerbation stage in the emergency department
}

\author{
Wei $\mathrm{Bi}^{1,2}$, Yan Sun ${ }^{3}$, Lin-qin $\mathrm{Ma}^{1,2}$, Cai-jun $\mathrm{Wu}^{1,2}$ \\ ${ }^{1}$ Department of Emergency, Beijing Dongzhimen Hospital, Beijing University of Chinese Medicine, Beijing 100700, China \\ ${ }^{2}$ Institute of Sepsis, Beijing University of Chinese Medicine, Beijing 100700, China \\ ${ }^{3}$ Department of Emergency, Sanhedongshan Hospital, Hebei 065200, China
}

Corresponding Author: Cai-jun Wu, Email:wucaijun@139.com

BACKGROUND: The study aimed to evaluate the predictive role of interleukin-6 (IL-6) and chronic obstructive pulmonary disease (COPD) assessment test (CAT) score in mechanical ventilation (MV) in COPD patients at the acute exacerbation stage in the emergency department (ED).

METHODS: For a one-year period, among adult patients in the ED who met the criteria of acute exacerbation of COPD, 158 who received MV within 48 hours after admission were compared to 294 who didn't require MV within the same period after admission. IL-6 level and CAT score were compared between the two groups. The predicted value of IL- 6 and CAT score was assessed by logistic regression analysis and a receiver operating characteristic (ROC) curve.

RESULTS: The IL-6 and CAT scores in the 158 MV patients were much higher than those without. IL-6 and CAT scores were independent predictors of MV within 48 hours using logistic regression analysis (IL-6: odds ratio [OR] 1.053, 95\% confidence interval [Cl] 1.039-1.067, $P<0.001$; CAT score: OR 1.122, 95\% Cl 1.086-1.159, $P<0.001$ ). The combination of IL-6 and CAT scores (area under ROC curve [AUC] $0.826,95 \% \mathrm{Cl} 0.786-0.866, P<0.001)$ improved the accuracy of predicting MV within 48 hours when compared with IL-6 (AUC 0.752, 95\% Cl 0.703-0.800, $P<0.001$ ) and CAT scores alone (AUC 0.739, $95 \% \mathrm{Cl} 0.692-0.786, P<0.001)$. The sensitivity and specificity were $69.6 \%, 74.1 \%, 75.32 \%$ and $63.6 \%$, respectively.

CONCLUSION : The combined of IL- 6 and CAT scores is useful for evaluating the risk of COPD patients at acute exacerbation in ED, and can provide a predictive value for MV or not within 48 hours.

KEY WORDS: Interleukin-6; Chronic obstructive pulmonary disease (COPD); COPD assessment test; Risk stratification; Receive operating characteristic curve

World J Emerg Med 2020;11(2):93-96

DOI: 10.5847/wjem.j.1920-8642.2020.02.005

\section{INTRODUCTION}

Chronic obstructive pulmonary disease (COPD) is a chronic airway inflammatory disease characterized by airflow limitation, with high morbidity and mortality. Acute exacerbation of COPD (AECOPD) can result in a sharp decline and deterioration of pulmonary function in patients with stable COPD.$^{[1]}$ The treatment cost at the acute phase is high and also takes up a large quantity of healthcare resources. Studies have shown that timely and appropriate treatment is beneficial for both improving therapeutic efficacy and prolonging long-term patient survival. ${ }^{[2]}$ As
AECOPD patients are usually first treated in the emergency department (ED), it is important for emergency physicians to accurately and rapidly evaluate the risks and to decide whether to use mechanical ventilation (MV) or not.

Interleukin-6 (IL-6) can increase collagen aggregation, inhibit the decomposition of extracellular matrix, and stimulate the proliferation of fibroblasts. ${ }^{[3]}$ This can lead to fibrous connective tissue formation and smooth muscle proliferation in the airway, and participation in airway remodeling regulation. Studies have shown that the levels of IL-6 in sputum, blood 
and bronchoalveolar lavage fluid in COPD patients are significantly increased at the acute exacerbation stage. ${ }^{[4,5]}$ The COPD Assessment Test (CAT) is a validated eightitem questionnaire designed to assess the effect of COPD symptoms on patient health status. In addition, it has been used to assess the severity of exacerbations and the health status of patients with AECOPD. It is also a potential indicator to assess the response to treatment. ${ }^{[6]}$ Both interleukin- 6 and CAT score have predictive value for AECOPD changes, and can be tested easily, but both of them can't be used as an evaluation criterion alone. Therefore, we presume that the combined use of interleukin- 6 and CAT score may improve sensitivity and clinical outcomes.

In this study we prospectively analyzed the IL-6 levels in AECOPD patients in emergency department, and assessed the predictive role of IL-6 levels with CAT score during MV.

\section{METHODS \\ Patients}

This was a prospective, observational clinical study. The patients were adults (age $\geq 18$ years old) who met the diagnostic criteria of AECOPD and visited the Department of Emergency Internal Medicine, Beijing Dongzhimen Hospital (Beijing, China) and Sanhedongshan Hospital (Hebei, China) between January 1 and December 31 in 2018. The AECOPD diagnostic criteria were in accordance with The Global Initiative for Chronic Obstructive Lung Disease of 2017. ${ }^{[7]}$ Exclusion criteria: (1) patients who died within 48 hours; (2) age $<18$ years old; (3) complications including pulmonary embolism; (4) diseases that seriously affectd short-term survival including congestive heart failure, severe liver and kidney dysfunction, metastatic cancer, and acquired immune deficiency syndrome; (5) patients or their agents didn't accept MV; (6) patients or their agents didn't agree to participate in the study; and (7) patients who had received treatment outside the hospital 24 hours before visit to our hospitals. The study abided by the medical ethics protocols and was approved by the hospital ethics committee. Patients or their agents signed the informed consent.

\section{Methods}

On the basis of routine medical examinations, 4 $\mathrm{mL}$ cubital fossa venous blood was collected in all enrolled patients within 2 hours after admission to our department for measurement of IL- 6 . The collected blood was centrifuged at 3,000 r/minute for 10 minutes and the serum was put in an antifreeze tube and stored at $-80^{\circ} \mathrm{C}$. During this time, CAT score of the patient was assessed by the attending physician. The eight items of the questionnaire included patient's symptoms, activities, psychology, sleep and social influence. No information was given to patients regarding score, and the questionnaire was to be finished by the patients themselves or with the aid of the agents. CAT score ranged from 0 to 40 points. The higher the score, the more severe the patient is affected by COPD. The white blood cell count (WBC) and partial pressure of carbon dioxide $\left(\mathrm{PCO}_{2}\right)$ in the blood gas analysis were also recorded during visits. The patients were followed up to 48 hours, and were divided into the ventilator treatment group and the non-ventilator treatment group according to whether they received MV or not. The IL-6 level and CAT scores were compared between the two groups.

\section{Statistical analysis}

Data was processed using SPSS 21.0 software. Normal distribution data was expressed as mean \pm standard deviation $(\bar{x} \pm s)$. Two independent sample tests were used for comparisons between groups. For enumeration data, chi-square test was used. Exact probability was used if the expected value was $<5$. The independent predictors of prognostic indexes were determined by logistic regression. The receiver operating characteristic curve (ROC curve) and area under the curve (AUC) was used to evaluate the prognostic value of independent predictors and to determine the cut-off level, sensitivity, specificity, positive predictive value, negative predictive value, positive likelihood ratio, and negative likelihood ratio. All tests were two-sided. $P<0.05$ was considered as statistically significant.

\section{RESULTS \\ Demographics of enrolled patients}

According to the inclusion and exclusion criteria of this study, 452 patients with AECOPD were enrolled. According to whether the patient received MV or not within 48 hours, they were divided into the ventilator treatment group $(n=158)$ and the non-ventilator treatment group $(n=294)$. The comparison of gender, age, $\mathrm{WBC}, \mathrm{PCO}_{2}$, IL- 6 and CAT scores within 2 hours was listed in Table 1 . The resluts of multi-factor logistic regression analysis of MV within 48 hours were presented in Table 2.

\section{Evaluation of MV within 48 hours using IL-6 level and the CAT scores}

The prediction accuracy of receiving MV within 48 hours by combined use of IL- 6 and the CAT scores (AUC $0.826,95 \%$ CI $0.786-0.866, P<0.001)$ was better than that by IL-6 alone (AUC $0.752,95 \%$ CI $0.703-0.800, P<0.001$ ) or the CAT score alone (AUC 0.739, 95\% CI 0.692-0.786, 
$P<0.001)$. The sensitivity and specificity were $69.6 \%$, $74.1 \%, 75.32 \%$ and $63.6 \%$ respectively (Figure 1).

\section{DISCUSSION}

AECOPD is one of the most common diseases encountered in the $\mathrm{ED}$, and is prone to worsening in the emergency treatment during which invasive or non-invasive MV is needed. Indications for invasive MV in COPD patients included the progressive worsening of respiratory failure, aggressive life-threatening acid-base imbalances, or altered consciousness after aggressive medication and noninvasive ventilation. The role of MV is to establish and manage artificial airways to effectively drain sputum, help to quickly control bronchial-pulmonary infections, and assume part or even the entire ventilation load to ensure effective ventilation of the body and at the same time allow the respiratory muscles to rest. ${ }^{[8,9]}$

Early risk assessment of MV in patients with emergency AECOPD is important to determine timely and correct treatment strategies. Studies have confirmed that the APACHE II score has a good predictive value for the prognosis of patients with AECOPD. ${ }^{[2,10]}$ However, the APACHE II score system is complicated and requires the input of clinical variables, from which a severity score is derived including age, heart rate, respiratory rate, mean

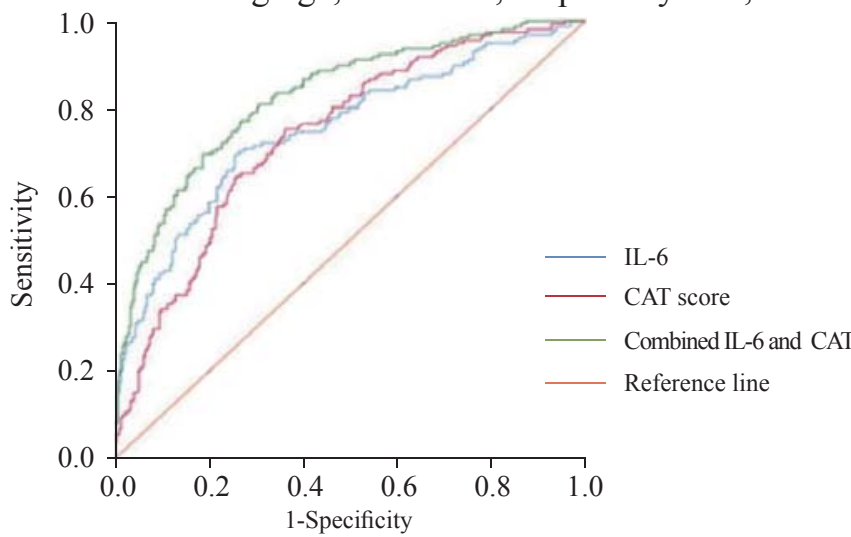

Figure 1. ROC curve analysis of the prognostic role of the CAT scores and IL-6 during MV. arterial pressure and temperature within 24 hours of admission. The CAT score is a short, simple questionnaire, for which the patient can finish in less than 5 minutes. The questionnaire, including the patient's symptoms, activity, psychology, sleep and social influence, covers a broad range of effects of COPD on patient health, with good measurement properties. Its simplicity and effectiveness have been confirmed by multiple-center clinical trials. ${ }^{[6]}$ Jones et $\mathrm{al}^{[11]}$ reported that the CAT correlates closely with healthrelated quality of life, as measured by the St George's Respiratory Questionnaire. There are no reports on CAT scores in emergency AECOPD patients to predict the risk of MV within 48 hours. In our study, we used IL-6 levels combined with CAT scores to determine the risk stratification of AECOPD patients during emergency visits, as well as the occurrence of ventilation events within 48 hours. We found that the CAT score in the ventilator treatment group significantly increased at the time of consultation compared with the non-ventilator treatment group, indicating that the patients in the ventilator treatment group had poor daily activities. Some previous indicators like white blood cell counts and lung function were regarded as "gold standard" for the diagnosis and evaluation of COPD patients' conditions, but they cannot fully reflect the overall physical function of patients with COPD. The sensitivity of the CAT score in assessing changes in the patient's condition is higher than that of the lung function tests, which provides a more convenient evaluation index for clinicians to judge the development of COPD in a timely and accurate manner. ${ }^{[12]} \mathrm{In}$ this study, the CAT score was used to evaluate the ventilation events in patients with emergency AECOPD within 48 hours, and it was found to have clinical predictive value (AUC 0.739, $P<0.001$ ).

We found that the blood concentration of IL- 6 in the ventilator treatment group was significantly higher than that in the non-ventilator treatment group, indicating the systemic inflammatory response in the ventilator treatment group was significantly worse than that of the non-ventilator treatment group. IL-6 is produced by both immune and non-immune

Table 1. Comparison of gender, age, WBC, $\mathrm{PCO}_{2}$, IL- 6 and CAT scores between the two groups

\begin{tabular}{|c|c|c|c|c|}
\hline Parameters & Ventilator treatment group $(n=158)$ & Non-ventilator treatment group $(n=294)$ & $t / \chi^{2}$ & $P$ \\
\hline Gender (male/female) & $114 / 44$ & $167 / 127$ & 10.295 & 0.001 \\
\hline Age (years old) & $67.06 \pm 12.04$ & $63.60 \pm 8.82$ & 3.18 & 0.046 \\
\hline $\mathrm{WBC}\left(\times 10^{9} / \mathrm{L}\right)$ & $12.91 \pm 4.23$ & $12.83 \pm 5.15$ & 0.166 & 0.057 \\
\hline $\mathrm{PCO}_{2}(\mathrm{mmHg})$ & $50.59 \pm 14.77$ & $48.21 \pm 15.36$ & 1.589 & 0.071 \\
\hline IL-6 (ng/L) & $122.93 \pm 21.34$ & $103.69 \pm 18.51$ & 9.98 & 0.012 \\
\hline CAT score (points) & $32.35 \pm 7.65$ & $25.29 \pm 8.12$ & 8.98 . & $<0.001$ \\
\hline
\end{tabular}

Table 2. Logistic regression analyses for MV in patients with AECOPD in 48 hours

\begin{tabular}{|c|c|c|c|c|}
\hline Parameters & Regression coefficient & Relative risk & OR 95\% CI & $P$ \\
\hline Gender & -0.651 & 0.522 & $0.315-0.522$ & 0.011 \\
\hline Age (years old) & 0.032 & 1.033 & $1.009-1.057$ & 0.007 \\
\hline IL-6 (ng/L) & 0.051 & 1.053 & $1.039-1.067$ & $<0.001$ \\
\hline CAT score & 0.115 & 1.122 & $1.086-1.159$ & $<0.001$ \\
\hline Constant & -10.692 & 0.000 & & $<0.001$ \\
\hline
\end{tabular}


cells, which can induce antigen-stimulated $\mathrm{T}$ lymphocyte proliferation and B lymphocyte maturation. This increases IgE secretion, which up-regulates the body's immune response, inducing a systemic inflammatory response. In addition, IL-6 can synergize with other cytokines to increase collagen aggregation to stimulate the proliferation of fibroblasts, leading to the formation of connective tissue in the respiratory tract. ${ }^{[13]}$ Studies have shown that the increase of serum IL-6 levels in AECOPD patients not only represents the body's defense against infection, but also can be used as an indicator of early inflammation in infection, which is significantly increased in acute inflammatory reactions. ${ }^{[14]}$ Chen et al ${ }^{[15]}$ believed that IL-6 was the best systemic inflammatory cytokine to reflect the dyspnea and pulmonary function. In this study, we found that there was a significant difference in the concentration of IL-6 between the two groups. However, there were no significant differences between the white blood cell count and the $\mathrm{PCO}_{2}$, which supported that IL-6 has certain advantages in the early prediction of AECOPD patients' condition. In this study, the IL-6 level was used to evaluate the need for MV in patients with emergency AECOPD within 48 hours, which had a clinical predictive value (AUC $0.752, P<0.001$ ). Thus, the combined use of CAT scores and IL- 6 levels presented a significant increase in the prediction accuracy of ventilation events in patients with emergency AECOPD within 48 hours of admission (AUC 0.826, $P<0.001$ ).

There are some limitations in this study. Firstly, the MV use of the enrolled AECOPD patients was inconsistent (initial or re-use). Secondly, due to the limited sample size, more factors that may affect the MV use were not included as variables (like previous underlying diseases). In addition, due to the different clinical indications for invasive and noninvasive ventilators, it may be more meaningful to further separate and analyze different ventilation events.

\section{CONCLUSION}

In conclusion, the combination of the CAT scoring system and circulating IL-6 levels can more accurately assess the risk of patients with $\mathrm{AECOPD}$, and has a predictive value for occurrence of ventilation events within 48 hours.

Funding: This study was supported by grants from Dongzhimen Hospital Fund of Special Talent (2018RC01) and from Beijing University of Chinese Medicine Fund of Project (2019-JYB-XJSJJ-025). Ethical approval: The study was approved by the hospital ethics committee.

Conflicts of interest: There is no conflict of interest.

Contributors: WB and YS contributed equally to the work. All authors contributed to the design, interpretation of the study and to further drafts.

\section{REFERENCE}

1 Zhu B, Wang Y, Ming J, Chen W, Zhang L. Disease burden of COPD in China: a systematic review. Int J Chron Obstruct Pulmon Dis. 2018;13:1353-64.

2 Steriade AT, Johari S, Sargarovschi N, Necula D, Tudose CE, Ionita $\mathrm{D}$, et al. Predictors of outcome of noninvasive ventilation in severe COPD exacerbation. BMC Pulm Med. 2019;19(1):131.

3 Chaouat A, Savale L, Chouaid C, Tu L, Sztrymf B, Canuet $\mathrm{M}$, et al. Role for interleukin-6 in COPD-related pulmonary hypertension. Chest. 2009;136(3):678-87.

4 Yang Y, Li W, Wang ZM, Sun GY, Zhou P, Han XL. Clinical significance of interleukin- 6 and -8 in patients with chronic periodontal disease and acute exacerbation of chronic obstructive pulmonary disease. Zhonghua Kou Qiang Yi Xue Za Zhi. 2018;53(5):312-7.

5 Zheng J, Shi Y, Xiong L, Zhang W, Li Y, Gibson PG, et al. The expression of IL-6, TNF- $\alpha$, and MCP-1 in respiratory viral infection in acute exacerbations of chronic obstructive pulmonary disease. J Immunol Res. 2017;2017:8539294.

6 Zhou A, Zhou Z, Peng Y, Zhao Y, Duan J, Chen P. The role of CAT in evaluating the response to treatment of patients with AECOPD. Int J Chron Obstruct Pulmon Dis. 2018;13: 2849-58.

7 Global Strategy for the Diagnosis, Management and Prevention of COPD, Global Initiative for Chronic Obstructive Lung Disease (GOLD) 2017. Available from: http://goldcopd.org.

8 Aloushan AF, Almoaiqel FA, Alghamdi RN, Alnahari FI, Aldosari AF, Masud N, et al. Assessment of knowledge, attitude and practice regarding oxygen therapy at emergency departments in Riyadh in 2017: A cross-sectional study. World J Emerg Med. 2019;10(2):88-93.

9 Arumugam SK, Mudali I, Strandvik G, EI-Menyar A, AI-Hassani A, AI-Thani H. Risk factors for ventilator-associated pneumonia in trauma patients: A descriptive analysis. World J Emerg Med. 2018;9(3):203-10.

10 Li S, Zheng YA. Correlation between procalcitonin and APACHE II score in patients with chronic obstructive pulmonary disease. Chin J Emerg Med. 2013;22(3):287-90.

11 Jones PW, Harding G, Berry P, Wiklund I, Chen WH, Kline Leidy N. Development and first validation of the COPD Assessment Test. Eur Respir J. 2009;34(3):648-54.

12 Chhabra SK, Dash DJ. Acute exacerbations of chronic obstructive pulmonary disease: causes and impacts. Indian J Chest Dis Allied Sci. 2014;56(2):93-104.

13 Chen L, Deng L, Zhao H, Huang X, He X, Li X, et al. Comparison of national early warning score, rapid emergency medicine score and acute physiology and chronic health evaluation II score for predicting outcome among emergency severe patients. Zhonghua Wei Zhong Bing Ji Jiu Yi Xue. 2017;29(12):1092-6.

14 Oh JY, Lee YS, Min KH, Hur GY, Lee SY, Kang KH, et al. Difference in systemic inflammation and predictors of acute exacerbation between smoking-associated COPD and tuberculosis-associated COPD. Int J Chron Obstruct Pulmon Dis. 2018;13:3381-7.

15 Chen YW, Leung JM, Sin DD. A systematic review of diagnostic biomarkers of copd exacerbation. PLoS One. 2016;11(7):e0158843.

Received September 23, 2019 Accepted after revision January 8, 2020 